Original article

\title{
The role of osteopontin in patients with type 2 diabetes and cognitive impairment
}

\author{
Maria V. Matveeva ${ }^{1}$, Yulia G. Samoilova ${ }^{1}$, Oksana A. Oleynik ${ }^{1}$, Olga S. Tonkikh ${ }^{1}$, \\ Daria V. Podchinenova ${ }^{1}$, Dmitry A. Kudlay ${ }^{2,3}$ \\ ${ }^{1}$ Siberian State Medical University, Tomsk, Russia \\ 2 Russian Academy of Sciences, Moscow, Russia \\ ${ }^{3}$ First Moscow State Medical University (Sechenov University), Moscow, Russia
}

Received 16 September 2021, Accepted 30 November 2021

(C) 2021, Russian Open Medical Journal

Abstract: Background - type 2 diabetes is associated with obesity and cardiovascular disease; in combination with dysmetabolic and proinflammatory pathophysiological mechanisms, it leads to cognitive impairment.

Objective - analysis of the osteopontin role in formation of cognitive disorders in patients with type 2 diabetes.

Material and Methods - the study complies with generally accepted ethical rules; it was approved by the Ethics Committee of Siberian State Medical University. It involved 50 patients with type 2 diabetes, who were divided into groups depending on the presence of cognitive impairment; the control group consisted of 25 subjects. All patients underwent general clinical examination, blood sampling for biochemical parameters, and plasma osteopontin content assessment. Magnetic resonance imaging (MRI) was performed on SIGNA Creator $E$ magnetic resonance imaging system, GE Healthcare, $1.5 \mathrm{~T}$, China. The employed techniques included dynamic contrast and arterial spin labeling, proton spectroscopy, tractography. SPSS Statistics software was used for statistical analysis.

Results - osteopontin levels were higher in patients with excess weight, hyperglycemia, hyperuricemia, dyslipidemia, and cognitive impairment; and in neuroimaging studies with microangiopathy, based on perfusion MRI, with impaired white matter integration, as well as with neurometabolism of choline, creatine and phosphocreatine metabolites in the hippocampus, as well as their NAA/Cr, NAA/Cho, Cho/Cr ratios $(p \leq 0.05)$.

Conclusion - patients with type 2 diabetes, along with cognitive and metabolic disorders, exhibited elevated levels of osteopontin, which was also associated with impaired cerebral vascularization in general, and white matter organization, as well as neurometabolism in the hippocampus.

Keywords: type 2 diabetes, osteopontin, magnetic resonance perfusion, magnetic resonance spectroscopy, cognitive impairment.

Cite as Matveeva MV, Samoilova YG, Oleynik OA, Tonkikh OS, Podchinenova DV, Kudlay DA. The role of osteopontin in patients with type 2 diabetes and cognitive impairment. Russian Open Medical Journal 2021; 10: e0408.

Correspondence to Maria V. Matveeva. Address: Department of Pediatric Diseases, Department of General Practice and Outpatient Therapy, Siberian State Medical University, 4 Moscovsky Tract, Tomsk 634050, Russia. Phone: +79138152552. E-mail: matveeva.mariia@yandex.ru.

\section{Introduction}

Diabetes mellitus is a common metabolic disorder, associated with chronic complications, including the development of cognitive impairments [1]. Despite the fact that cognitive impairment is manifested in mild to moderate form, it can significantly impede daily activities, negatively affecting the quality of life [2]. It is well known that type 2 diabetes is a disease associated with obesity, insulin resistance, dyslipidemia and high blood pressure, leading to unfavorable cardiovascular outcomes $[3,4]$. All of such changes are considered factors of inflammation, which leads to metabolic disorders, including those in the central nervous system [5, 6]. One of promising cytokines regulating proinflammatory pathways in patients with type 2 diabetes and obesity is osteopontin, the change in which is also independently associated with a combined endpoint of adverse cardiovascular outcomes, as well as with hospitalization rates for heart failure [6, 7]. Besides, it was shown that osteopontin can affect both vascular and neurodegenerative dementia [8].
The study of functioning and metabolic changes in the brain, associated with dysmetabolic and proinflammatory mechanisms, is possible due to contemporary neuroimaging techniques, which allow assessing regional neurochemical profiles (proton magnetic resonance spectroscopy), topological integration of the white matter in the brain (tractography), along with the features of microcirculation in the presence of microangiopathy (perfusion magnetic resonance imaging [MRI]) [9-11].

The hypothesis: in patients with type 2 diabetes and cognitive impairment, a higher level of osteopontin in plasma is observed, associated with changes in neurometabolism and neurovascularization of the brain.

\section{Material and Methods}

All procedures performed in studies involving human participants were in compliance with the ethical standards of the institutional research committee and with 1964 Declaration of 
Helsinki and its later amendments, or comparable ethical standards. The study was approved by the ethics committee of the Federal State Budgetary Institution of Higher Education Siberian State Medical University of the Ministry of Healthcare of Russia (hereinafter referred to as SibMed) (Protocol No. 5265 of February 05, 2017); all subjects have signed informed consent for participation in this one-stage continuous study. The exclusion criteria were: other types of diabetes (type 1 diabetes or gestational diabetes), organic brain diseases, psychiatric diseases, contraindications to MRI, glomerular filtration rate less than 60 $\mathrm{mL} / \mathrm{min}$, and severe vision and hearing loss.

By simple randomization, 45-65 years old patients with type 2 diabetes of varying duration of the disease were distributed among two groups: Group 1 included the patients with type 2 diabetes without cognitive impairment $(n=25)$, Group 2 encompassed subjects with type 2 diabetes and cognitive impairment $(n=25)$. The control group (Group 3) consisted of 25 healthy volunteers with matching ages and genders.

In all patients, anthropometric parameters were measured, including height, weight, and BMI. Screening for cognitive disorders was performed using the Montreal Cognitive Assessment Scale (MoCA test). The degree of cognitive impairment was established in strict accordance with conventionally accepted criteria sensu the classification by Academician of the Russian Academy of Medical Sciences N.N. Yakhno (2005), identifying severe, moderate and mild grades of the cognitive impairment.

All patients underwent blood sampling for glycosylated hemoglobin (HbA1c), fasting plasma glucose, alkaline phosphatase, total bilirubin, alanine aminotransferase (ALT), aspartate aminotransferase (AST), urea, creatinine with assessment of the glomerular filtration rate (GFR), total uric acid, high-density lipoproteins (HDL), low-density lipoproteins (LDL), triglycerides, and osteopontin. These indicators were assessed at the central research laboratory of SibMed.

MRI was performed on the SIGNA Creator E magnetic resonance imaging system, GE Healthcare, 1.5 T, China. Dynamic contrast MRI was used with imaging, weighted by the inhomogeneity of the magnetic field (dynamic susceptibility contrast MR perfusion), and the technique of arterial spin labeling (ASL), which did not require the introduction of a contrast agent and allowed quantitative assessment of cerebral blood flow. The contrast substance was Gadobutrol, administered intravenously in a bolus dose of $5 \mathrm{~mL}$. The following parameters were used for tractography: $\mathrm{TR}=1000 \mathrm{~ms}, \mathrm{TE}=\mathrm{min}, \mathrm{FOV}=240 \times 240$, image matrix of $96 \times 96$ with subsequent interpolation up to $256 \times 256$, slice thickness of $2.5 \mathrm{~mm}$, distance between slices of $0 \mathrm{~mm}, \mathrm{NEX}=1$. Scanning was performed in coronary projection. A single volume was obtained with a value of the diffusion factor $b=0,120$ volumes were taken with different isotropically distributed directions of the diffusion gradient at $b=3000 \mathrm{~s} / \mathrm{mm}^{2}$. Data processing was carried out using the FSL software (FMRIB Software Library v. 5.0, Oxford, UK, http://fsl.fmrib.ox.ac.uk/fsl/fslwiki); the construction of brain tracts was conducted by using the Explore DTI program (http://www.exploredti.com). Proton magnetic resonance spectroscopy of the brain was performed without changing the apparatus and the position of the subject's body, with a relaxation time $T E=135 \mathrm{~ms}$, the volume of one voxel of $1.5 \mathrm{~cm}^{3}$, in a multivoxel mode.

The main spectra of $\mathrm{N}$-acetylaspartate (NAA), choline (Cho), creatine $(\mathrm{Cr})$, and creatine phosphate $(\mathrm{PCr})$ metabolites and their ratios were recorded in the gray matter of the cerebral cortex, white matter of the brain, subcortical structures, and in the hippocampus on both sides (left and right).

Table 1. Characteristics of patients with type 2 diabetes and in the control group, Me (Q1-Q3)

\begin{tabular}{|c|c|c|c|}
\hline Characteristics & Group 1 & Group 2 & Group 3 (control) \\
\hline Age, years & $62.6(58.5-67.5)$ & $62.2(58.0-67.0)$ & $62.6(59.0-67.0)$ \\
\hline Height, $\mathrm{cm}$ & $171.0(164.0-179.0)$ & $169.6(163.0-175.0)$ & $169.1(163.0-178.0)$ \\
\hline Weight, kg*,** & $84.0(76.1-91.3)$ & $98.6(84.5-109.0)$ & $70.0(59.0-78.0)$ \\
\hline $\mathrm{BMI} *, * *$ & $28.5(25.7-31.7)$ & $35.0(29.7-39.4)$ & $24.7(23.0-26.3)$ \\
\hline $\mathrm{HbA1c}, \% *, * *$ & $7,7(7.3-8.6)$ & $8.6(7.5-9.0)$ & $4.9(4.6-5.3)$ \\
\hline Fasting glucose, $\mu \mathrm{mol} / \mathrm{L}, \mathrm{mmol} / \mathrm{L} * * *$ & $7.6(6.8-8.4)$ & $8.8(7.5-9.1)$ & $4.8(4.2-5.3)$ \\
\hline Alkaline phosphatase, units/L** & $84.0(63.5-98.0)$ & $85.2(69.0-95.0)$ & $54.4(45.0-63.0)$ \\
\hline Total bilirubin, $\mu \mathrm{mol} / \mathrm{L}$ & $11.1(8.5-14.0)$ & $11.3(8.0-14.0)$ & $10.3(8.0-13.0)$ \\
\hline ALT, units/L & $43.4(25.0-52.0)$ & $44.3(25.5-53.5)$ & $22.5(19.0-25.0)$ \\
\hline AST, units/L & $33.5(19.0-44.5)$ & $33.4(19.0-44.0)$ & $24.4(23.0-28.0)$ \\
\hline Urea, $\mu \mathrm{mol} / \mathrm{L}$ & $6.5(5.0-7.7)$ & $6.0(4.9-7.0)$ & $5.7(4.2-6.7)$ \\
\hline Creatinine, $\mu \mathrm{mol} / \mathrm{L}$ & $77.1(65.0-93.0)$ & $77.8(64.0-93.0)$ & $81.1(69.0-92.0)$ \\
\hline Uric acid, $\mu \mathrm{mol} / \mathrm{L}^{* *}$ & $344.7(277.0-380.5)$ & $349.7(279.0-423.0)$ & $225.7(194.0-264.0)$ \\
\hline $\mathrm{GFR}, \mathrm{mL} / \mathrm{min} / 1.73 \mathrm{~m}^{2}$ & $82.3(71.5-95.5)$ & $87.6(74.0-106.0)$ & $77.8(71.0-89.0)$ \\
\hline $\mathrm{HDL}, \mu \mathrm{mol} / \mathrm{L}^{* *}$ & $1,2(1.0-1.3)$ & $1,2(1.0-1.3)$ & $1.6(1.4-1.7)$ \\
\hline $\mathrm{LDL}, \mu \mathrm{mol} / \mathrm{L} * *$ & $3.5(2.9-4.3)$ & $3.7(3.0-4.3)$ & $1.6(1.2-1.8)$ \\
\hline Triglycerides, $\mu \mathrm{mol} / \mathrm{L}^{* *}$ & $2.7(1.7-3.3)$ & $2.8(2.1-3.4)$ & $1.4(1.1-1.9)$ \\
\hline Total cholesterol, $\mu \mathrm{mol} / \mathrm{L} *, * *$ & $5.5(4.7-6.4)$ & $6.5(6.0-7.3)$ & $4.6(4.0-5.2)$ \\
\hline Osteopontin, ng/mL $*, * *$ & $0.2(0.1-0.2)$ & $0.3(0.1-0.4)$ & $0.0(0.0-0.1)$ \\
\hline MoCA test $* * *$ & $28.5(28.0-29.5)$ & $22.2(20.0-24.0)$ & $29.0(28.0-30.0)$ \\
\hline
\end{tabular}

* Group 1 vs. Group 2, $p \leq 0.05$; ** Group 2 vs. Group 3, $p \leq 0.05$. BMI, body mass index; HbA1c, glycosylated hemoglobin; ALT, alanine aminotransferase; AST, aspartate aminotransferase; GFR, glomerular filtration rate; HDL, high-density lipoproteins; LDL, low-density lipoproteins; MoCA, Montreal Cognitive Assessment Scale. 
Table 2. Correlation analysis of osteopontin level and fractional anisotropy during brain tractography

\begin{tabular}{lcc}
\hline Tracts & $\begin{array}{c}\text { Correlation coefficient } \\
\text { (Spearman), } R\end{array}$ & $p$ \\
\hline Right corticospinal tract & -0.443 & 0.00006 \\
\hline Right corticospinal tract & -0.283 & 0.01 \\
\hline $\begin{array}{l}\text { Uncinate fasciculus (hook-shaped } \\
\text { bundle of axons) on the left side }\end{array}$ & -0.2658 & 0.02 \\
\hline Knee of the corpus callosum & -0.249 & 0.03 \\
\hline
\end{tabular}

Table 3. Correlation analysis of osteopontin level and metabolites of the brain proton spectroscopy

\begin{tabular}{lcc}
\hline $\begin{array}{l}\text { Metabolites } \\
\text { and their ratios }\end{array}$ & $\begin{array}{c}\text { Correlation coefficient } \\
\text { (Spearman), } R\end{array}$ & $p$ \\
\hline Cho left & 0.544 & 0.0001 \\
Cr right & 0.490 & 0.000008 \\
PCr left & 0.247 & 0.03 \\
PCr on right & 0.410 & 0.0002 \\
NAA/Cr left & 0.472 & 0.00001 \\
NAA/Cr right & 0.517 & 0.000002 \\
NAA/Cho left & 0.337 & 0.003 \\
Cho/Cr left & 0.300 & 0.008 \\
Cho/Cr right & -0.461 & 0.00003 \\
\hline
\end{tabular}

Cho, choline; $\mathrm{Cr}$, creatine; $\mathrm{PCr}$, phosphocreatine; NAA, $\mathrm{N}$-acetylaspartate.

For statistical analysis, the SPSS Statistics software was used for frequency analysis, Kendall rank correlation coefficient for samples that do not comply with the law of normal distribution, Kruskal-Wallis nonparametric analysis of variance for comparing the sample medians; $p$-values less than 0.05 were considered indicative of statistically significant dependencies.

\section{Results}

The analysis yielded the differences between the groups of patients with type 2 diabetes, with or without cognitive disorders, compared with the control group, in the following parameters: weight, BMI, HbA1C, fasting glucose, alkaline phosphatase, ALT, uric acid, HDL, LDL, triglycerides, total cholesterol, osteopontin, and results of the MoCA test (Table 1 ).

The study revealed the positive correlation between the osteopontin content and excess weight $(R=0.459, p=0.00003), B M I$ $(R=0.459, p=0.00003), \mathrm{HbA1c}(\mathrm{R}=0.467, p=0.00002)$, fasting plasma glucose $(R=0.459, p=0.00003)$, uric acid $(R=0.397, p=0.00004)$, $L D L$ $(R=0.388, p=0.00005)$, triglycerides $(R=0.330, p=0.003)$, and total cholesterol ( $R=0.447, p=0.00005)$. The negative correlation was established between osteopontin and HDL content $(-R=0.386$, $p=0.00007)$, and with the MoCA score $(-R=0.613, p=0.00001)$.

Further on, we analyzed the relationship between osteopontin and brain neurovascularization, which was changed in the areas of frontal, parietal, occipital, and temporal lobes of white and gray matter, accumbens nucleus, left head of the caudate nucleus, and globus pallidus in terms of the mean transit time of blood flow (MTT, sec) sensu contrast perfusion, and of total cerebral blood flow sensu non-contrast imaging ( $p \leq 0.05)$.

When assessing the brain tractography, we discovered a decrease in the fractional anisotropy of the tracts in patients with type 2 diabetes with cognitive impairment and increased level of osteopontin (Table 2).

The data of the statistical study on metabolites sensu proton brain spectroscopy demonstrated a relationship between the osteopontin content and several parameters: choline, creatine and phosphocreatine in the hippocampus, and also their ratios, $\mathrm{NAA} / \mathrm{Cr}$, NAA/Cho, Cho/Cr (Table 3).

\section{Discussion}

Data from systematic reviews (of 24 and 27 studies) verified the association of type 2 diabetes with cognitive impairment, the risk factors for which are decompensation of glycemic control, disease duration, and the presence of microvascular complications $[12,13]$. Indeed, in our study, patients with type 2 diabetes and cognitive impairment had hyperglycemia, hypercholesterolemia, obesity, and an increased level of osteopontin. Obesity and type 2 diabetes are associated with endothelial dysfunction and dysregulation of vascular homeostasis, which could contribute to microangiopathy, including cerebral vessels, with the deposition of osteopontin (a cell adhesion protein of the extracellular matrix produced by visceral adipose tissue). The latter could affect vascular calcification, as well as neuroinflammation [14, 15].

It should be noted that the level of this marker in blood plasma increases in insulin-resistant conditions, such as obesity and type 2 diabetes, and is also associated with an increased risk of cardiovascular events [16]. For example, early studies demonstrated a negative relationship between osteopontin level, total cholesterol and LDL in serum, which suggested that hypercholesterolemia promoted vascular calcification by suppressing the osteopontin synthesis [17]. On the other hand, in patients with type 2 diabetes in our study, a positive association between osteopontin content and total cholesterol may reflect some protective mechanism against the dysmetabolic increase of vascular calcification. In another study, tissue markers of cholesterol synthesis rather than plasma were associated with osteopontin level in carbohydrate metabolic disorder [18].

It was shown that osteopontin can act as an activator of microglia in hypoxic brain lesions [20]. That is, probably, why the perfusion studies of the central nervous system in patients with type 2 diabetes and cognitive impairment revealed an association with changes in the neurovascularization of the general cerebral blood flow and regions, responsible for impaired motor and executive functions, attention and memory (frontal, parietal, occipital and temporal lobes of white and gray matter, accumbens nucleus, left head of caudate nucleus, and globus pallidus), which is typical for this type of diabetes [21].

Besides, hippocampus region is of particular importance in the context of dementia in type 2 diabetes, when changes in neurometabolism are noted even before any clinical manifestation [22]. This study revealed a change in the level of osteopontin and other indicators, such as choline, creatine and phosphocreatine (the main participants in the metabolism of cell membranes and energy transport), in the hippocampus, as well as their ratios: $\mathrm{NAA} / \mathrm{Cr}$, NAA/Cho, Cho/ $\mathrm{Cr}$ in the hippocampus. These changes lead to cellular patterns of osteopontin introduction in reactive glial cells, suggesting that this marker plays a multifunctional role in the pathogenesis of ischemic damage in type 2 diabetes [23]. Also, the data of brain tractography, when there was a decrease in the fractional anisotropy of white matter with an increased osteopontin level, highlighted the vascular genesis of cognitive impairment development in type 2 diabetes [24]. At the same time, a decrease in anisotropy in the corpus callosum region may also be a predictor of cognitive impairment in type 2 diabetes. 


\section{Conclusion}

In patients with type 2 diabetes, cognitive disorder and metabolic impairment (obesity, dyslipidemia, symptoms of nonalcoholic fatty liver disease), an increased level of osteopontin was recorded. The latter is associated with impaired metabolism, functioning and vascularization of the entire brain and its areas responsible for cognitive functions.

\section{Conflict of Interest}

The authors declare that they have no conflicts of interest.

\section{Funding}

This study was supported by the Presidential Grant, Agreement No. 075-15-2020-192 of March 19, 2020.

\section{References}

1. Muriach M, Flores-Bellver M, Romero FJ, Barcia JM. Diabetes and the brain: Oxidative stress, inflammation, and autophagy. Oxid Med Cell Longev 2014; 2014: 102158. https://doi.org/10.1155/2014/102158.

2. Sinclair AJ, Girling AJ, Bayer AJ. Cognitive dysfunction in older subjects with diabetes mellitus: Impact on diabetes self-management and use of care services. Diabetes Res Clin Pract 2000; 50(3): 203-212. https://doi.org/10.1016/s0168-8227(00)00195-9.

3. Eckel RH, Grundy SM, Zimmet PZ. The metabolic syndrome. Lancet 2005; 365(9468): 1415-1428. https://doi.org/10.1016/s01406736(05)66378-7.

4. Gaede $P$, Vedel $P$, Larsen N, Jensen GV, Parving $H H$, Pedersen $O$. Multifactorial intervention and cardiovascular disease in patients with type 2 diabetes. N Engl J Med 2003; 348(5): 383-393. https://doi.org/10.1056/nejmoa021778.

5. Cai D, Liu T. Inflammatory cause of metabolic syndrome via brain stress and NF-KB. Aging (Albany NY) 2012; 4(2): 98-115. https://doi.org/10.18632/aging.100431.

6. Matveeva MV, Samoilova YuG, Zhukova NG, Oleynik OA, Rotkank MA. Tauopathy and cognitive impairment in experimental diabetes mellitus. Diabetes Mellitus 2017; 20(3): 181-184. Russian. https://doi.org/10.14341/2072-0351-5842.

7. Kahles F, Findeisen HM, Bruemmer D. Osteopontin: A novel regulator at the cross roads of inflammation, obesity and diabetes. Mol Metab 2014; 3(4): 384-393. https://doi.org/10.1016/j.molmet.2014.03.004.

8. Abdalrhim AD, Marroush TS, Austin EE, Gersh BJ, Solak N, Rizvi SA, et al. Plasma osteopontin levels and adverse cardiovascular outcomes in the PEACE trial. PLoS One 2016; 11(6): e0156965. https://doi.org/10.1371/journal.pone.0156965

9. Chai $Y L$, Chong JR, Raquib AR, Xu X, Hilal S, Venketasubramanian N, et al. Plasma osteopontin as a biomarker of Alzheimer's disease and vascular cognitive impairment. Sci Rep 2021; 11(1): 4010. https://doi.org/10.1038/s41598-021-83601-6.

10. Duarte JM. Metabolic alterations associated to brain dysfunction in diabetes. Aging Dis 2015; 6(5): 304-321. https://doi.org/10.14336/ad.2014.1104.

11. Kim DJ, Yu JH, Shin MS, Shin YW, Kim MS. Hyperglycemia Reduces efficiency of brain networks in subjects with type 2 diabetes. PLoS One 2016; 11(6): e0157268. https://doi.org/10.1371/journal.pone.0157268.

12. Vancheri F, Longo G, Vancheri S, Henein M. Coronary microvascular dysfunction. J Clin Med 2020; 9(9): 2880. https://doi.org/10.3390/jcm9092880.

13. van den Berg E, Kloppenborg RP, Kessels RPC, Kappelle LJ, Biessels GJ. Type 2 diabetes mellitus, hypertension, dyslipidemia and obesity: A systematic comparison of their impact on cognition. Biochim Biophys
Acta
2009;
1792(5):
$470-481$

https://doi.org/10.1016/j.bbadis.2008.09.004.

14. Palta $P$, Schneider ALC, Biessels GJ, Touradji $P$, Hill-Briggs F. Magnitude of cognitive dysfunction in adults with type 2 diabetes: A meta-analysis of six cognitive domains and the most frequently reported neuropsychological tests within domains. J Int Neuropsychol Soc 2014 20(3): 278-291. https://doi.org/10.1017/s1355617713001483.

15. Barton M. Obesity and aging: Determinants of endothelial cell dysfunction and atherosclerosis. Pflugers Arch 2010; 460(5): 825-837. https://doi.org/10.1007/s00424-010-0860-y.

16. Moorman HR, Poschel D, Klement JD, Lu C, Redd PS, Liu K. Osteopontin: A key regulator of tumor progression and immunomodulation. Cancers (Basel) 2020; 12(11): 3379. https://doi.org/10.3390/cancers12113379.

17. Schinzari F, Tesauro M, Bertoli A, Valentini A, Veneziani A, Campia U, et al. Calcification biomarkers and vascular dysfunction in obesity and type 2 diabetes: Influence of oral hypoglycemic agents. Am J Physio Endocrinol Metab 2019; 317(4): E658-E666. https://doi.org/10.1152/ajpendo.00204.2019.

18. Takemoto M, Tada K, Nakatsuka K, Moriyama Y, Kazui H, Yokote K, et al. Effects of aging and hyperlipidemia on plasma osteopontin level. Nihon Ronen Igakkai Zasshi 1999; 36(11): 799-802. Japanese. https://doi.org/10.3143/geriatrics.36.799.

19. Luomala M, Päivä $H$, Thelen K, Laaksonen R, Saarela M, Mattila K, et al. Osteopontin levels are associated with cholesterol synthesis markers in mildly hypercholesterolemic patients. Acta Cardiol 2007; 62(2): $177-$ 181. https://doi.org/10.2143/ac.62.2.2020239.

20. Remmerie A, Martens L, Thoné T, Castoldi A, Seurinck R, Pavie B, et al. Osteopontin expression identifies a subset of recruited macrophages distinct from Kupffer cells in the fatty liver. Immunity 2020; 53(3): 641657.e14. https://doi.org/10.1016/i.immuni.2020.08.004.

21. Li Y, Dammer EB, Zhang-Brotzge $X$, Chen S, Duong DM, Seyfried NT, et al. Osteopontin is a blood biomarker for microglial activation and brain injury in experimental hypoxic-ischemic encephalopathy. eNeuro 2017 4(1): ENEURO.0253-16.2016. https://doi.org/10.1523/eneuro.025316.2016.

22. Zilliox LA, Chadrasekaran K, Kwan JY, Russell JW. Diabetes and cognitive impairment. Curr Diab Rep 2016; 16(9): 87. https://doi.org/10.1007/s11892-016-0775-x.

23. Samoilova J, Matveeva M, Tonkih O, Kudlau D, Oleynik O, Kanev A. A prospective study: Highlights of hippocampal spectroscopy in cognitive impairment in patients with type 1 and type 2 diabetes. J Pers Med 2021; 11(2): 148. https://doi.org/10.3390/jpm11020148.

24. Lee JY, Choi JS, Choi JY, Shin YJ, Yun H, Cha JH, et al. Spatial and temporal changes of osteopontin in oxygen-glucose-deprived hippocampal slice cultures. Acta Neurobiol Exp (Wars) 2010; 70(1): 1 12. https://pubmed.ncbi.nlm.nih.gov/20407481.

25. Grand Moursel L, van der Graaf LM, Bulk M, van Roon-Mom WMC, van der Weerd L. Osteopontin and phospho-SMAD2 / 3 are associated with calcification of vessels in D-CAA, a hereditary cerebral amyloid angiopathy. Brain Pathol 2019; 29(6): 793-802. https://doi.org/10.1111/bpa.12721.

\section{Authors:}

Maria V. Matveeva - MD, PhD, Associate Professor, Department of Pediatric Diseases, Department of General Practice and Outpatient Therapy, Siberian State Medical University, Tomsk, Russia. https://orcid.org/0000-0001-9966-6686.

Yulia G. Samoylova - MD, PhD, Professor, Chair of the Department of Pediatric Diseases, Department of Faculty Therapy with a Course in Clinical Pharmacology, Siberian State Medical University, Tomsk, Russia. https://orcid.org/0000-0002-2667-4842.

Oksana A. Oleynik - PhD, Associate Professor, Department of Faculty Therapy with a Course in Clinical Pharmacology, Siberian State Medical University, Tomsk, Russia. https://orcid.org/0000-0002-2482-8393. 
Olga S. Tonkikh - PhD, Chair of the Department of Tomographic Methods, Siberian State Medical University, Tomsk, Russia. https://orcid.org/00000003-0589-0260.

Daria V. Podchinenova - PhD, Associate Professor, Department of Pediatric Diseases, Siberian State Medical University, Tomsk, Russia. https://orcid.org/0000-0001-6212-4568.

Dmitry A. Kudlay - MD, PhD, Professor, Lead Researcher, Laboratory of Personalized Medicine and Molecular Immunology No. 71, State Research Center for Immunology, Russian Academy of Sciences, Moscow, Russia; Professor, Department of Pharmacology, Institute of Pharmacy, I.M. Sechenov First Moscow State Medical University (Sechenov University), Moscow, Russia. https://orcid.org/0000-0003-1878-4467. 